\title{
Fast Water Flow during the Hydration/Dehydration of Montmorillonite
}

\author{
TuAN A. Ho, * YifENG WANG, CARLOS JOVE-COLON
}

Sandia National Laboratories, Albuquerque, New Mexico

87185, USA (*taho@sandia.gov)

Smectite clays including Na-montmorillonite (Na-MMT) are phyllosilicates or sheet silicate minerals that can internally adsorb water at interlayers causing the clay $\mathrm{d}(001)$ (interlayer) spacing to increase from $\sim 9.6 \AA$ (dehydrated) to $\sim 12.5 \AA$ (monohydrated), and to $\sim 15 \AA$ (bihydrated). However, the mechanism for water movement (e.g., Darcy flow, molecular diffusion) through the interlayer during hydration/dehydration is not well understood. Using molecular dynamics simulations we investigate the water transport through a bihydrated Na-MMT channel (Fig. 1). When both left- and right-hand side reservoirs in Fig. 1 are fully occupied by water molecules and a pressure of $10 \mathrm{MPa}$ is applied to the left "piston", there is almost no net water transport through the interlayer channel. However, when we evaporate the water at the right-hand side reservoir water

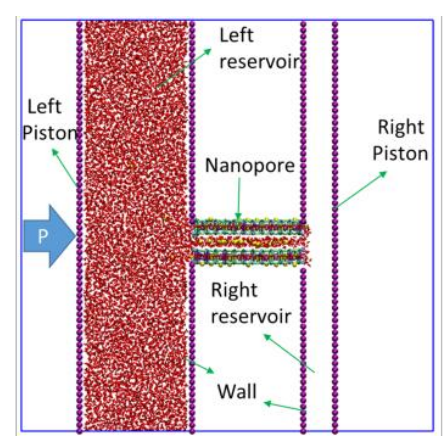

Fig. 1 Simulation system used to study the water transport through Na-MMT nanopore molecules move through the nanochannel with unexpectedly large velocity $(\sim 0.88 \mathrm{~m} / \mathrm{s})$. The water transport through the channel can be modeled using a modified Darcy's law that includes a pressure threshold to accurately describe the behavior that flow rate in the clay interlayer is insensitive to the external mechanical pressure. The results also suggest that the flow rate depends on the meniscus position controlled by the evaporation process. The flow rate is enhanced when the meniscus forms at the large $\mathrm{OH}$-functionalized edge surfaces. Our results provide an insight into the water transport through the clay interlayer during the hydration/dehydration processes.

Sandia National Laboratories is a multi-mission laboratory managed and operated by National Technology and Engineering Solutions of Sandia, LLC., a wholly owned subsidiary of Honeywell International, Inc., for the U.S. Department of Energy's National Nuclear Security Administration under contract DE-NA0003525. 